\title{
A discrete methodology for controlling the sign of curvature and torsion for NURBS
}

\author{
Alexandros I. Ginnis · E. I. Karousos • P. D. Kaklis
}

Received: 29 June 2009 / Accepted: 13 July 2009 / Published online: 7 August 2009

(C) Springer-Verlag 2009

\begin{abstract}
This paper develops a discrete methodology for approximating the so-called convex domain of a NURBS curve, namely the domain in the ambient space, where a user-specified control point is free to move so that the curvature and torsion retains its sign along the NURBS parametric domain of definition. The methodology provides a monotonic sequence of convex polyhedra, converging from the interior to the convex domain. If the latter is non-empty, a simple algorithm is proposed, that yields a sequence of polytopes converging uniformly to the restriction of the convex domain to any user-specified bounding box. The algorithm is illustrated for a pair of planar and a spatial Bézier configuration.
\end{abstract}

Keywords Curves $\cdot$ Curvature $\cdot$ Torsion $\cdot$ NURBS $\cdot$ Knot insertion

Mathematics Subject Classification (2000) $\quad 65 \mathrm{D} 15 \cdot 68 \mathrm{U} 07$

\section{Introduction}

Differential geometry invariants under reparameterization, such as the curvature and torsion of curves, constitute important features in the creation, analysis and modification of shape. In order to secure robustness and achieve a satisfactory level of efficiency when handling such shape features, the user should possess an accurate and easily computable quantification of their dependence with respect to the degrees of freedom provided by the adopted geometry representation.

Communicated by C. H. Cap.

A. I. Ginnis $(\varangle)$ · E. I. Karousos · P. D. Kaklis

School of Naval Architecture and Marine Engineering, National Technical University of Athens,

Athens, Greece

e-mail: ginnis@naval.ntua.gr 
This paper focuses on the sign of curvature and torsion of planar and spatial NURBS, respectively. More specifically, our aim is to quantify the dependence of the sign of these invariants with respect to the control points, appearing in the NURBS representation. This quantification should provide accurately described feasibility domains in the ambient space, where the chosen control points are free to move without altering the sign of the invariant over a subinterval of the NURBS parametric domain of definition. A CAD system may exploit this knowledge to improve its services both on the interactive and automatic level. Interactivity can be enhanced in two ways:

- If the designer aims to retain the sign of the invariant, the system constrains the control point movement in the interior of the feasibility domain.

- If the designer seeks to change the sign of the invariant, the system provides the shortest path direction for minimizing shape variation.

On the other hand, improvement on the automatic level may include the functionality of finding the optimal, against a fairness criterion, location of the control points in the feasibility domain.

The herein adopted approach for constructing the feasibility domain for a user-specified control point is to combine NURBS basis properties (Sect. 3) with preliminary results (Sect. 2) from a generic methodology developed by the authors, that addresses the problem of controlling the sign of curvature [5], and torsion [6], for the class of parametric curves that adopt the control point paradigm. Eventually, we get a methodology that provides a monotonic sequence of convex polyhedra, converging from the interior to the feasibility domain; see Theorem 1. Being convex, the feasibility domain will be henceforth referred to as the convex domain. In the case it is non empty, the methodology of Sect. 3 yields a simple algorithm presented and illustrated in Sect. 4 for a pair of planar and a spatial Bézier configuration.

\section{Generic problem and preliminary results}

Suppose that a family of parametric curves is given by

$$
\mathbf{F}(\mathbf{d} ; t)=\mathbf{d} \cdot N(t)+\mathbf{s}(t),
$$

which maps $\mathbf{d} \in \mathbb{E}^{n}, n=2,3$, to a curve with $t$ ranging in a compact interval $I \subset \mathbb{R}$ and $N: I \longrightarrow \mathbb{R}, \mathbf{s}: I \longrightarrow \mathbb{R}^{n}$ being two sufficiently differentiable functions. The problem to be investigated is which members of $\mathbf{F}(\mathbf{d} ; t)$ are regular curves of constant sign of curvature for $n=2$ or torsion for $n=3$. Curve families as in (1) can naturally stem from curves whose parametric representation adopts the control point paradigm with all control points kept fixed but one, say d, that will be referred to as the free control point. Let us start with

Definition 1 Let be given a family of parametric curves $\mathbf{F}(\mathbf{d} ; t), t \in I, \mathbf{d} \in \mathbb{E}^{n}, n=2,3$ and a fixed $t_{0} \in I$.

1. For $n=2$, the domain of positive curvature $D_{\kappa+}\left(t_{0}\right)$ of $\mathbf{F}(\mathbf{d} ; t)$ with respect to $t_{0}$ consists of all possible locations of $\mathbf{d}$ for which $\mathbf{F}\left(\mathbf{d} ; t_{0}\right)$ is an ordinary point of positive curvature. 
2. For $n=3$, the domain of positive torsion $D_{\tau+}\left(t_{0}\right)$ of $\mathbf{F}(\mathbf{d} ; t)$ with respect to $t_{0}$, consists of all possible locations of $\mathbf{d}$ for which $\mathbf{F}\left(\mathbf{d} ; t_{0}\right)$ is an ordinary point of non-zero curvature and positive torsion.

The domains $D_{\kappa-}\left(t_{0}\right)$ and $D_{\tau-}\left(t_{0}\right)$ can be analogously defined.

To deal with the curvature-sign of planar curves we first set $\mathbf{d}=(x, y), \mathbf{F}=\left(F_{1}, F_{2}\right)$, $\mathbf{s}=\left(s_{1}, s_{2}\right)$, and recall that the sign of curvature at a regular point of a planar curve coincides with the sign of the quantity

$$
h_{t}(x, y)=\dot{F}_{1} \ddot{F}_{2}-\ddot{F}_{1} \dot{F}_{2}
$$

where dot signifies differentiation with respect to $t$. Setting

$$
a_{1}(t)=\left|\begin{array}{ll}
\dot{N}(t) & \ddot{N}(t) \\
\dot{s}_{2}(t) & \ddot{s}_{2}(t)
\end{array}\right|, \quad a_{2}(t)=\left|\begin{array}{cc}
\dot{s}_{1}(t) & \ddot{s}_{1}(t) \\
\dot{N}(t) & \ddot{N}(t)
\end{array}\right|, \quad b(t)=\left|\begin{array}{ll}
\dot{s}_{1}(t) & \ddot{s}_{1}(t) \\
\dot{s}_{2}(t) & \ddot{s}_{2}(t)
\end{array}\right|,
$$

(2) can be written as

$$
h_{t}(x, y)=a_{1}(t) x+a_{2}(t) y+b(t) \text {. }
$$

To do the analogous job for the torsion sign of spatial curves we set $\mathbf{d}=(x, y, z)$, $\mathbf{F}=\left(F_{1}, F_{2}, F_{3}\right)$ and $\mathbf{s}=\left(s_{1}, s_{2}, s_{3}\right)$. Then, the sign of torsion at a regular and non-zero curvature point coincides with the sign of the quantity

$$
\mathcal{H}_{t}(x, y, z):=[\dot{\mathbf{F}}, \ddot{\mathbf{F}}, \dddot{\mathbf{F}}]
$$

where $[\mathbf{a}, \mathbf{b}, \mathbf{c}]$ denotes the standard triple scalar product of vectors $\mathbf{a}, \mathbf{b}$ and $\mathbf{c}$. Setting

$$
a_{1}(t):=\left|\begin{array}{ccc}
\dot{N} & \ddot{N} & \dddot{N} \\
\dot{s_{2}} & \ddot{s_{2}} & \dddot{s_{2}} \\
\dot{s_{3}} & \ddot{s_{3}} & \ddot{s_{3}}
\end{array}\right|, a_{2}(t):=\left|\begin{array}{ccc}
\dot{s_{1}} & \ddot{s_{1}} & \dddot{s_{1}} \\
\dot{N} & \ddot{N} & \ddot{N} \\
\dot{s_{3}} & \ddot{s_{3}} & \ddot{s_{3}}
\end{array}\right|, a_{3}(t):=\left|\begin{array}{ccc}
\dot{s_{1}} & \ddot{s_{1}} & \dddot{s_{1}} \\
\dot{s_{2}} & \ddot{s_{2}} & \dddot{s_{2}} \\
\dot{N} & \ddot{N} & \ddot{N}
\end{array}\right|, b(t):=\left|\begin{array}{lll}
\dot{s}_{1} & \ddot{s_{1}} & \dddot{s_{1}} \\
\dot{s_{2}} & \ddot{s_{2}} & \dddot{s_{2}} \\
\dot{s_{3}} & \ddot{s_{3}} & \ddot{s_{3}}
\end{array}\right|,
$$

(5) can be written as

$$
\mathcal{H}_{t}(x, y, z)=a_{1}(t) x+a_{2}(t) y+a_{3}(t) z+b(t) .
$$

The following preliminary results establish that $D_{\kappa+}\left(t_{0}\right)$ and $D_{\tau+}\left(t_{0}\right)$ are, in general, half-planes/-spaces, respectively.

\section{Proposition 1}

1. Let $n=2$ and $a_{i}(t), i=1,2$, be defined as in (3). If $\mathbf{c}_{\kappa}=\left(a_{1}\left(t_{0}\right), a_{2}\left(t_{0}\right)\right) \neq \mathbf{0}$, then the domains of positive, $D_{\kappa+}\left(t_{0}\right)$, and negative, $D_{\kappa-}\left(t_{0}\right)$, curvature with respect to $t_{0}$ are the two open half-planes bounded by the line $h_{t_{0}}(x, y)=0 ;$ for a detailed proof see [5]. 
2. Let $n=3$ and $a_{i}(t), i=1,2,3$, be defined as in (6). If $\mathbf{c}_{\tau}=\left(a_{1}\left(t_{0}\right), a_{2}\left(t_{0}\right)\right.$, $\left.a_{3}\left(t_{0}\right)\right) \neq \mathbf{0}$, then the domains of positive, $D_{\tau+}\left(t_{0}\right)$, and negative, $D_{\tau-}\left(t_{0}\right)$, torsion with respect to $t_{0}$ are the two open half-spaces bounded by the half-plane $\mathcal{H}_{t_{0}}(x, y, z)=0 ;$ for a detailed proof see [6].

In view of the non-vanishing hypothesis on the quantities $\mathbf{c}_{\kappa}$ and $\mathbf{c}_{\tau}$, appearing in the above proposition, it is natural to inquire what is happening when this condition is not met. This issue is clarified in the below proposition.

Proposition 2 Let $\bullet=\kappa / \tau \pm$ and $\mathbf{c}_{\kappa / \tau}=\mathbf{0}$. Then:

(i) If $\dot{N}\left(t_{0}\right) \neq 0$, then $D_{\bullet}\left(t_{0}\right)$ is empty or contains exactly one point.

(ii) If $\dot{N}\left(t_{0}\right)=0$ and $\dot{\mathbf{s}}\left(t_{0}\right) \neq \mathbf{0}$, then $D_{\bullet}\left(t_{0}\right)$ is either empty or coincides with the ambient space $\mathbb{E}^{n}, n=2,3$.

(iii) If $\dot{N}\left(t_{0}\right)=0$ and $\dot{\mathbf{s}}\left(t_{0}\right)=\mathbf{0}$ then, to reach a conclusion about $D_{\bullet}\left(t_{0}\right)$, one needs to identify the first non-vanishing higher-order derivatives of these quantities.

Proof Without loss of generality we shall treat only the planar case.

(i) Since $a_{i}\left(t_{0}\right)=0, i=1,2$, and $\dot{N}\left(t_{0}\right) \neq 0$, the first two of formulae (3) lead to

$$
\ddot{\mathbf{s}}_{i}\left(t_{0}\right)=\dot{\mathbf{s}}_{i}\left(t_{0}\right) \frac{\ddot{N}\left(t_{0}\right)}{\dot{N}\left(t_{0}\right)}, \quad i=1,2,
$$

i.e., $\dot{\mathbf{s}}_{i}\left(t_{0}\right)$ and $\ddot{\mathbf{s}}_{i}\left(t_{0}\right)$ are linearly dependent which, in view of the third formula in (3), gives that $b\left(t_{0}\right)$ and thus $h_{t_{0}}(x, y)$ (see Eq. 2) vanishes at any location $\mathbf{d}=(x, y)$ in $\mathbb{E}^{2}$. This would imply that the curvature $\kappa\left(t_{0}\right)$ vanishes as well, provided that the curve $\mathbf{F}(\mathbf{d} ; t)$ is regular at $t=t_{0}$. Using (1) it is easy to show that this is indeed the case for all

$$
\mathbf{d} \neq \mathbf{d}^{\star}=-\frac{\dot{s}\left(t_{0}\right)}{\dot{N}\left(t_{0}\right)} .
$$

Summarizing, curvature $\kappa\left(t_{0}\right)$ vanishes for $\mathbf{d} \neq \mathbf{d}^{\star}$ and, as a consequence, all $\mathbf{d} \neq \mathbf{d}^{\star}$ cannot belong to $D_{\kappa \pm}\left(t_{0}\right)$. Now, if $\mathbf{d}=\mathbf{d}^{\star}$ then $\mathbf{F}\left(\mathbf{d}^{\star} ; t\right)$ is not anymore regular at $t=t_{0}\left(\dot{\mathbf{F}}\left(\mathbf{d}^{\star} ; t_{0}\right)=\mathbf{0}\right)$ and one cannot deduce general conditions guaranteeing that $\kappa\left(t_{0}\right)$ is definable. If $\kappa\left(t_{0}\right)$ can be eventually defined, by identifying the first non-vanishing higher derivatives of $h_{t}\left(\mathbf{d}^{\star}\right)$ and $\dot{\mathbf{F}}\left(\mathbf{d}^{\star} ; t\right)$ as $t \rightarrow t_{0}$, and possesses the desired sign, then $D_{\kappa \pm}\left(t_{0}\right)$ will contain only one element, namely $\mathbf{d}^{\star}$. Otherwise, $D_{\kappa \pm}\left(t_{0}\right)$ will be empty.

(ii) In this case $\mathbf{F}\left(\mathbf{d} ; t_{0}\right)$ is regular for every $\mathbf{d} \in \mathbb{E}^{2}$ and thus the sign of $\kappa\left(t_{0}\right)$ is equal to that of $b\left(t_{0}\right)$. If $b\left(t_{0}\right)$ has the desired sign then $D_{\kappa \pm}\left(t_{0}\right)$ coincides with $\mathbb{E}^{2}$, otherwise it is the empty set.

(iii) In this case $\mathbf{F}\left(\mathbf{d} ; t_{0}\right)$ is non-regular while $h_{t_{0}}(x, y)$ vanishes for all $\mathbf{d} \in \mathbb{E}^{2}$. Consequently, one cannot deduce general conditions that guarantee definability of $\kappa\left(t_{0}\right)$.

Henceforth, and without loss of generality, we shall deal with the problem of maintaining positive curvature or torsion not at a single parametric point, $t_{0}$, but over the 
whole interval $I$. Extending Definition 1 we denote by $D_{\bullet}(I), \bullet=\kappa / \tau$, the domain of positive curvature or torsion of a family of curves $\mathbf{F}(\mathbf{d} ; t), t \in I$, i.e., the set of all possible locations of $\mathbf{d}$ for which $\mathbf{F}(\mathbf{d} ; t)$, defined as in (1), has positive curvature or torsion over $I$. Noting that

$$
D_{\bullet}(I)=\bigcap_{t \in I} D_{\bullet}(t)=K \cap L, \quad K=\bigcap_{\substack{t \in I \\ \mathbf{c}_{\bullet} \neq \mathbf{0}}} D_{\bullet}(t), \quad L=\bigcap_{\substack{t \in I \\ \mathbf{c}_{\bullet}=\mathbf{0}}} D_{\bullet}(t),
$$

Proposition 1 implies that the sets

$$
K_{\kappa}=\left\{(x, y) \in \mathbb{E}^{2}: h_{t}(x, y)=a_{1}(t) x+a_{2}(t) y+b(t)>0, t \in I, \mathbf{c}_{\kappa} \neq \mathbf{0}\right\}
$$

and

$$
K_{\tau}=\left\{(x, y, z) \in \mathbb{E}^{3}: \mathcal{H}_{t}(x, y, z)=a_{1}(t) x+a_{2}(t) y+a_{3}(t) z+b(t)>0, t \in I, \mathbf{c}_{\tau} \neq \mathbf{0}\right\}
$$

are convex, being the intersection of a one-parameter family of open half-planes/spaces, respectively. The sets $K_{\kappa}, K_{\tau}$ will be collectively referred to as the convex domains. In order to get a complete representation of $D_{\bullet}(I)$, papers [5] and [6] focused on developing methodologies for expressing the convex domains as finite intersections of not necessarily linear but easily computable convex sets.

As for $L$, viewing Proposition 2 in the context of the most popular versions of the control-point paradigm, it is easy to see that $L$ can be expressed as the intersection of finite, possibly degenerate ( $\varnothing$ or $\mathbb{E}^{n}, n=2,3$, ) sets, and in this connection its computation is left to be done on a case-by-case basis.

\section{Polyhedral approximation of the convex domain via knot insertion}

As pointed out in the introduction, this work aims to develop a discrete method for approximating the convex domains $K_{\bullet}, \bullet=\kappa / \tau$, when the curve family $\mathbf{F}(\mathbf{d} ; t)$ is, with respect to $t \in I$, a NURBS curve. Without loss of generality the proposed approach will be presented in detail for the planar case, namely approximating $K_{\kappa}$. Nevertheless, its generalization for the spatial domain $K_{\tau}$ is natural and will be numerically illustrated, along with $K_{\kappa}$, in Sect. 3 as well.

In the planar case, the functions $a_{i}(t), i=1,2$, and $b(t)$, appearing in the righthand side of (4), will be NURBS functions, as a result of the fact that they are expressed as products of the NURBS functions $\dot{N}(t), \ddot{N}(t), \dot{s}_{i}(t)$ and $\ddot{s}_{i}(t), i=1,2$; see (3). To find the NURBS representation of a product of NURBS, one may appeal to a direct algorithmic approach, proposed in [8] and adopted herein, which supports symbolic computation of the coefficients of the product after finding the knot vector of the product. Alternatively, in case the previous approach is computationally expensive and complex to implement, one might choose to exploit the $B$-spline representation 
uniqueness property and compute the coefficients of the product by solving an equivalent interpolation problem; see [1].

In the NURBS setting introduced above, the inequality in the definition (11) of $K_{\kappa}$ can be written as

$$
\sum_{j=0}^{p}\left(a_{1 j} x+a_{2 j} y+b_{j}\right) N_{j q}(t)>0, \quad t \in I,
$$

where $\left\{N_{j q}(t)\right\}_{j=0}^{p}$ is the common NURBS basis of order $q$ and knot vector $\mathcal{T}=$ $\left\{t_{0}, \ldots, t_{p+q}\right\}$, with respect to which the NURBS functions $a_{i}(t), i=1,2$, and $b(t)$ are being expressed. Exploiting the non-negativity of $N_{j q}(t)$, one can get from (13) the following finite set of discrete inequalities

$$
a_{1 j} x+a_{2 j} y+b_{j}>0, \quad j=0, \ldots, p .
$$

The above inequality set provides a sufficient condition for fulfilling (13). In geometric terms, (14) determines a polygonal subset, say $K_{\kappa, d i s c r}$, of the sought-for convex domain $K_{\kappa} . K_{\kappa, \text { discr }}$ is a convex polygon obtained by intersecting the $p+1$ half-planes in (14). Then, the usefulness of working with (14) instead of (13) depends on whether $K_{\kappa, \text { discr }}$ is close enough to $K_{\kappa}$, in other words, how far from being necessary is the inequality set (14). Towards this aim, we shall exploit the well known property of NURBS that the corners of their control polygon converge quadratically to the curve as knot spacing goes to zero. So, after applying repeatedly simultaneous knot insertion in $a_{i}(t), i=1,2$, and $b(t)$, (13) takes the following form

$$
\sum_{j=0}^{p_{\ell}}\left(a_{1 j}^{(\ell)} x+a_{2 j}^{(\ell)} y+b_{j}^{(\ell)}\right) N_{j q}^{(\ell)}(t)>0, t \in I, \quad p_{\ell}=p+\lambda 2^{\ell-1}, \ell=1,2, \ldots,
$$

where $\lambda$ denotes the number of non-degenerate subintervals occurring in the original knot vector $\mathcal{T}$, while $\left\{N_{j q}^{(\ell)}(t)\right\}$ is the basis obtained from the $(\ell-1)$-level basis, $\left\{N_{j q}^{(\ell-1)}(t)\right\}$, by knot insertion at the midpoints of the non-degenerate knot subintervals of its knot vector $\mathcal{T}^{(l-1)}$; obviously: $N_{j q}^{(0)}(t):=N_{j q}(t)$ and $\mathcal{T}^{(0)}:=\mathcal{T}$. In direct analogy with the pair (13) and (14), inequality (15) gives the following set of discrete inequalities

$$
a_{1 j}^{(\ell)} x+a_{2 j}^{(\ell)} y+b_{j}^{(\ell)}>0, \quad j=0, \ldots, p_{\ell} .
$$

Let $K_{\kappa, \text { discr }}^{(\ell)}$ be the convex polygon defined by (16). We now state and prove the following result, establishing the convergence of $K_{\kappa, \text { discr }}^{(\ell)}$ towards $K_{\kappa}$ as $\ell$ increases.

Theorem $1 \forall\left(x_{0}, y_{0}\right) \in K_{\kappa}, \exists \ell_{0}:\left(x_{0}, y_{0}\right) \in K_{\kappa, \text { discr }}^{(\ell)}$ for $\ell \geq \ell_{0}$. 


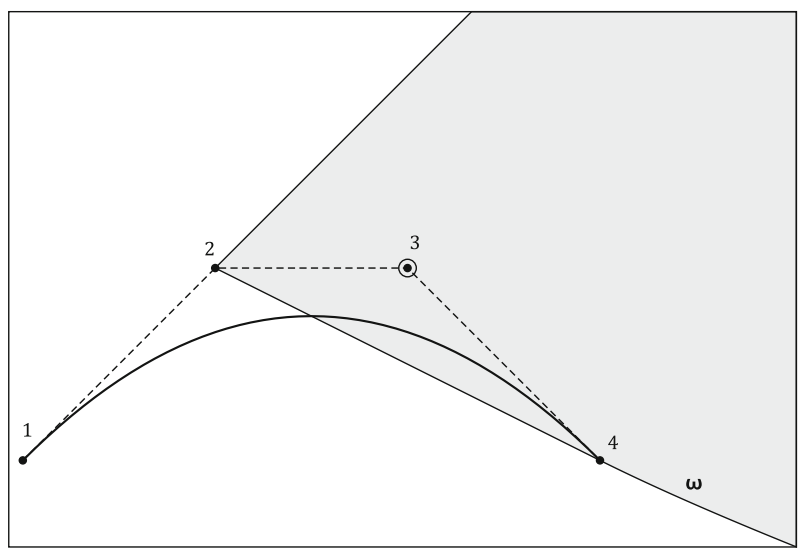

Fig. 1 A cubic planar Bézier curve with the free control point $\mathbf{d}$ depicted by a circled bullet. A part of the unbounded convex domain $K_{\kappa}$ is depicted in gray. Part $\omega$ of $\partial K_{K}$, emanating from the control point nr.4 and tending to infinity, is non-linear

Proof Since $\left(x_{0}, y_{0}\right)^{T} \in K_{\kappa}$, the function

$$
h_{t}\left(x_{0}, y_{0}\right)=a_{1}(t) x_{0}+a_{2}(t) y_{0}+b(t), \quad t \in I,
$$

is a positive NURBS function for $t \in I$. Then, appealing to the convergence property of the knot insertion process, we can say that there will be an index $\ell_{0}$ such that, for all $\ell \geq \ell_{0}$, the vertices $\mathbf{v}_{j}^{(\ell)}, j=0, \ldots, p_{\ell}$, of the control polygon of $h_{t}\left(x_{0}, y_{0}\right)$, and thus the control polygon itself, will entirely lie in the positive half-plane. Recalling that

$$
\mathbf{v}_{j}^{(\ell)}\left(x_{0}, y_{0}\right)=\left(g_{j}^{(\ell)}, a_{1 j}^{(\ell)} x_{0}+a_{2 j}^{(\ell)} y_{0}+b_{j}^{(\ell)}\right), \quad j=0, \ldots, p_{\ell},
$$

with $g_{j}^{(\ell)}$ denoting the Greville abscissae (see $\S 8.6$ in [2]), we conclude that $a_{1 j}^{(\ell)} x_{0}+$ $a_{2 j}^{(\ell)} y_{0}+b_{j}^{(\ell)}$ will be positive for $\ell \geq \ell_{0}$, i.e., $\left(x_{0}, y_{0}\right)$ will lie in $K_{\kappa, d i s c r}^{(\ell)}$ for $\ell \geq \ell_{0}$.

Remark 1 Theorem 1 guarantees the pointwise convergence of $K_{\kappa, \text { discr }}^{(\ell)}$ to $K_{\kappa}$ for large $\ell$. In order to investigate the question of the uniformity of convergence, we assume that $(x, y)$ lies in a compact subset $\mathcal{K}_{\kappa}$ of $K_{\kappa}$. Then, in more quantitative terms, we recall the below given bound (see Lemma 4.1 in Chapter 4 of [7]), which reveals that, via repeated simultaneous knot insertion, the control coefficients $c_{i}$ of a spline function $f$ of order $q$ converge quadratically to the function values at the Greville abscissae:

$$
\left|c_{i}-f\left(g_{i}\right)\right| \leq \mathcal{C}\left(t_{i+q-1}-t_{i+1}\right)^{2}\left\|D^{2} f\right\| .
$$

Here $\|\cdot\|$ denotes the max-norm, $g_{i}=\left(t_{i+1}+\cdots+t_{i+q-1}\right) /(q-1)$ are the Greville abscissae, $D^{2}$ denotes (one-sided) differentiation (from the right) and, finally, the constant $\mathcal{C}$ depends only on $q$. Applying (18) for the family of positive NURBS functions 

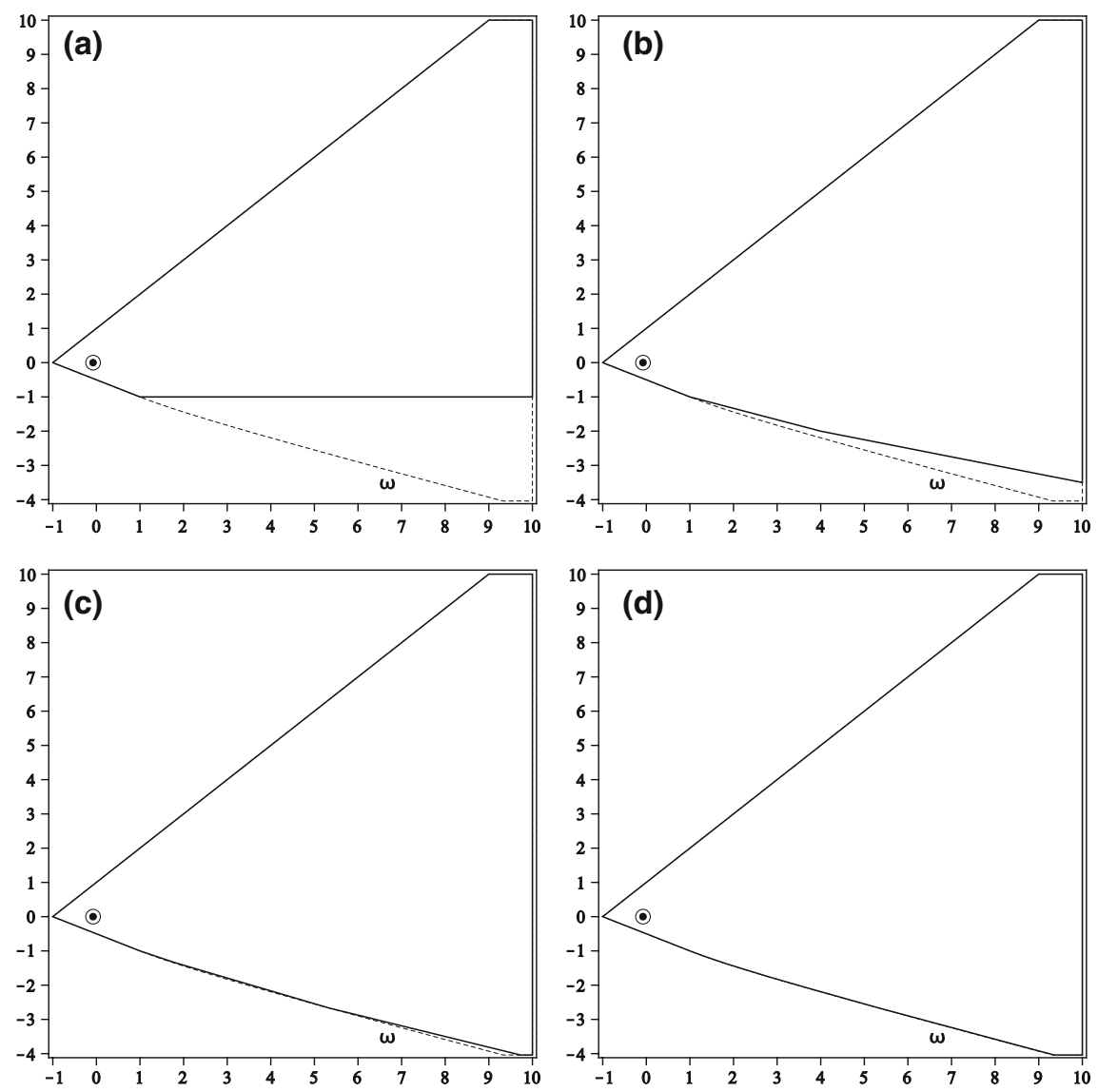

Fig. 2 Convex polytopes $K_{\kappa, \text { discr }}^{(\ell)}$ (solid line) calculated at iteration level: a $\ell=0, \mathbf{b} \ell=1$, c $\ell=2$, d $\ell=4 . K_{\kappa}$ is depicted by dashed line

Fig. 3 A quintic planar Bézier curve with the free control point d depicted by a circled bullet. $K_{\kappa}$ is a triangle, part of which is depicted in gray

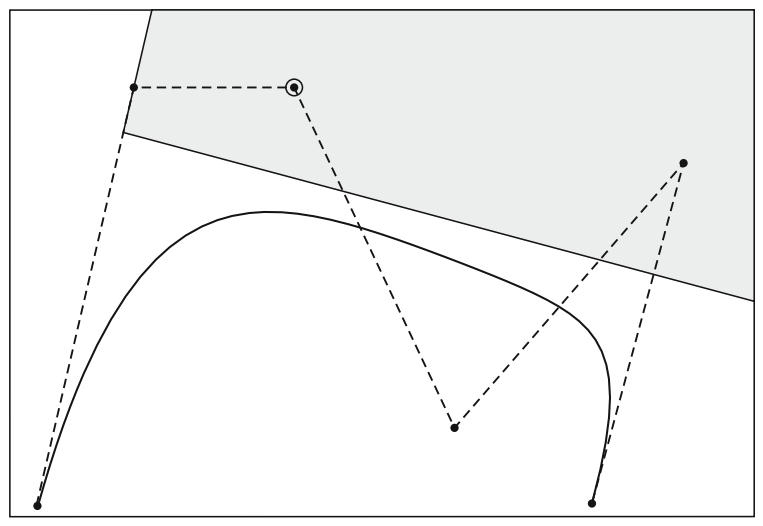



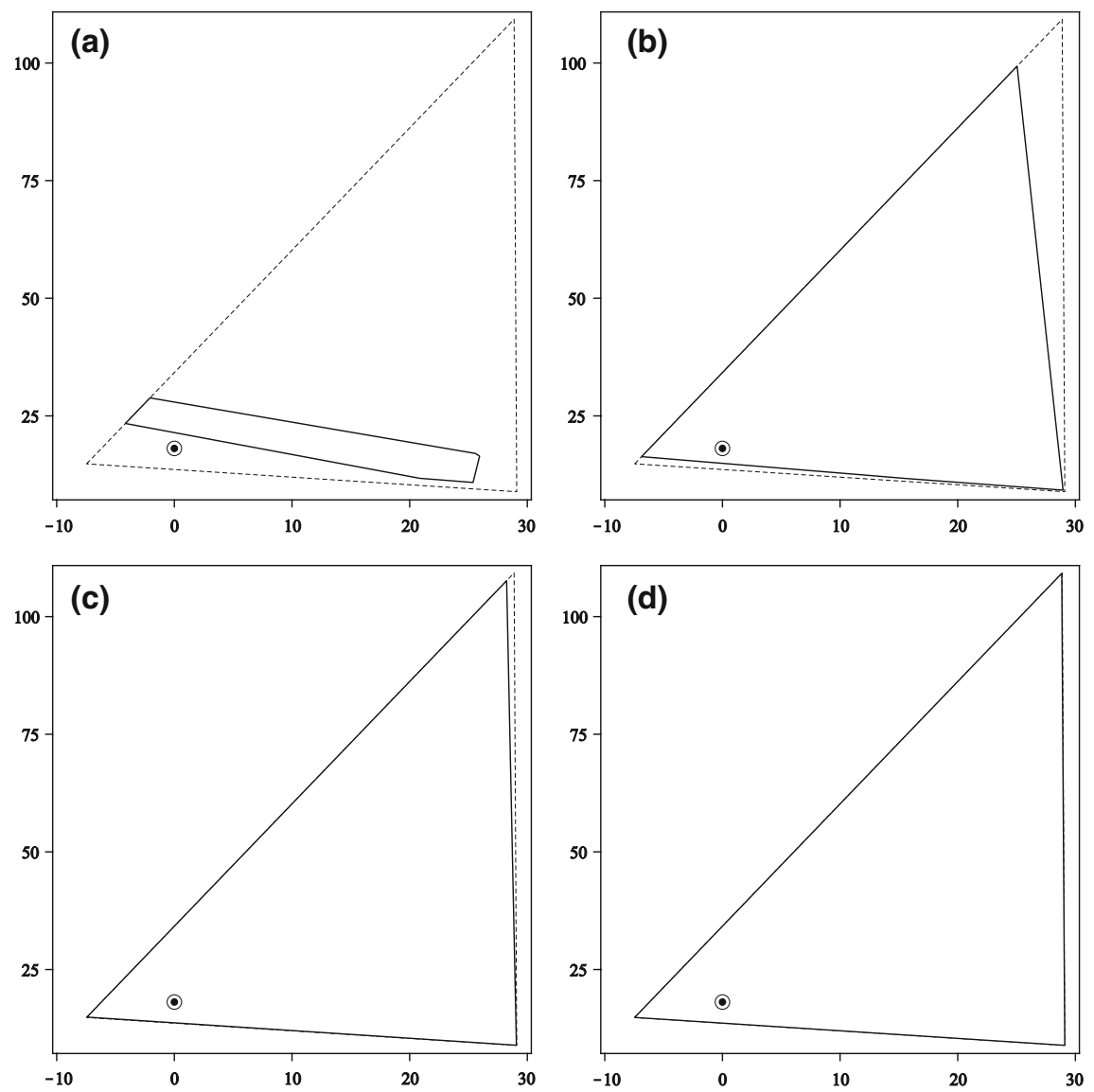

Fig. 4 Convex polytopes $K_{\kappa, \text { discr }}^{(\ell)}$ (solid line) calculated at iteration level: a $\ell=1, \mathbf{b} \ell=2, \mathbf{c} \ell=4$, $\mathbf{d} \ell=6 . K_{K}$ is depicted by dashed line

$f(t)=h_{t}(x, y)$ with $(x, y) \in \mathcal{K}_{\kappa}$, it is readily seen that, since $\left\|D^{2} f\right\|$ can be bounded by an $(x, y)$-independent constant, uniform convergence over $\mathcal{K}_{\kappa}$ can be proved by employing the argumentation of Theorem 1.

Remark 2 The sequence of the convex polygons $K_{\kappa, \text { discr }}^{(\ell)}$ is monotone in the following sense: $K_{\kappa, \text { discr }}^{(\ell)} \subseteq K_{\kappa, \text { discr }}^{(\ell+1)}$. To prove this property, let $\left(x_{0}, y_{0}\right) \in K_{\kappa, \text { discr }}^{(\ell)}$, which implies that the control points $\mathbf{v}_{j}^{(\ell)}\left(x_{0}, y_{0}\right)$ lie in the positive half-plane. Then, since knot insertion is a corner cutting process, it becomes obvious that $\mathbf{v}_{j}^{(\ell+1)}\left(x_{0}, y_{0}\right)$ will also lie in the positive half-plane and thus $\left(x_{0}, y_{0}\right) \in K_{\kappa, \text { discr }}^{(\ell+1)}$ as well.

\section{A discrete algorithm for non-empty convex domains}

Based on the methodology presented in the previous section, we can readily propose an algorithm for approximating the convex domain $K_{\kappa}$ in the case it is not empty. 


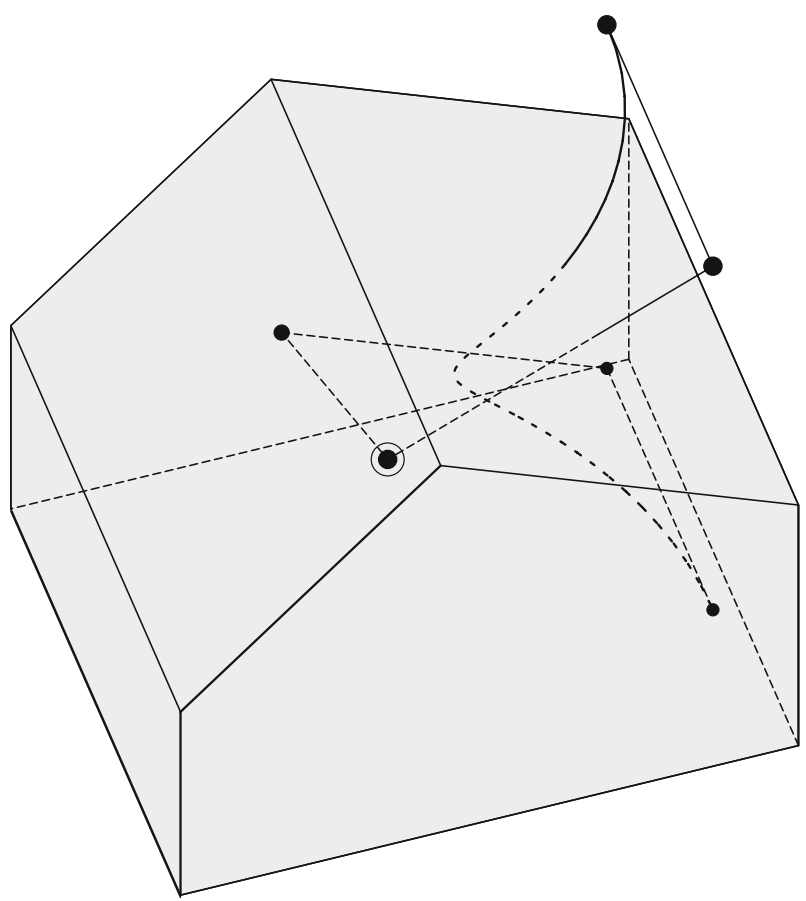

Fig. 5 A quintic spatial Bézier curve with the free control point $\mathbf{d}$ depicted by a circled bullet. A part of the unbounded convex domain $K_{\tau}$ is depicted in gray

(a)
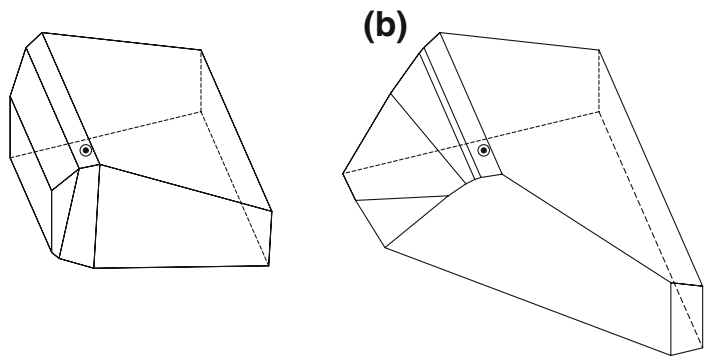

(c)

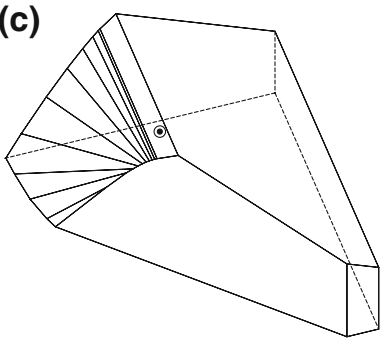

(d)

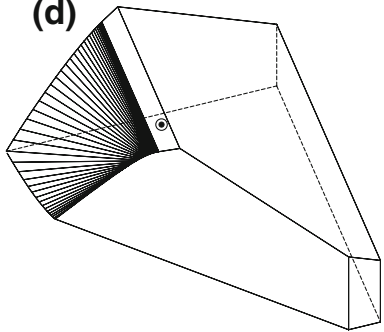

Fig. 6 Convex polytopes $K_{\tau, \text { discr }}^{(\ell)}$ (solid line) calculated at iteration level: a $\ell=0, \mathbf{b} \ell=1$, c $\ell=2$, d $\ell=4$ 
The algorithm is iterative, providing at each step $\ell$ a convex polytope $K_{\kappa, \text { discr }}^{(\ell)}$. The so resulting sequence of polytopes converges uniformly to the restriction of $K_{\kappa}$ to any bounding box $\mathcal{B}$, as guaranteed by Remark 1 . Convergence is controlled through the symmetric difference, also called Nikodym metric, $\delta(A, B)$ for convex bodies, which is expressed as the area of the symmetric difference of $A$ and $B$ [4]. Since, however, the sequence $\left\{K_{\text {discr }}^{(\ell)}\right\}$ is monotone (see Remark 2), we have

$$
\delta\left(K_{\kappa, \text { discr }}^{(\ell+1)}, K_{\kappa, \text { discr }}^{(\ell)}\right)=\operatorname{area}\left(K_{\kappa, \text { discr }}^{(\ell+1)} \backslash K_{\kappa, \text { discr }}^{(\ell)}\right)
$$

The algorithm adopts the relative error implied by (19), namely

$$
\text { rel.error }\left(K_{\kappa, \text { discr }}^{(\ell+1)}, K_{\kappa, \text { discr }}^{(\ell)}\right)=\operatorname{area}\left(K_{\kappa, \text { discr }}^{(\ell+1)} \backslash K_{\kappa, \text { discr }}^{(\ell)}\right) / \operatorname{area}\left(K_{\kappa, \text { discr }}^{(\ell)}\right)
$$

and terminates, after a finite number of steps, when rel.error gets smaller than a user-specified tolerance parameter $\mathrm{tol}$. We are now ready to state the algorithm:

\section{Algorithm APPROXIMATECONVEXDOMAIN}

Input. An initial NURBS curve, the free control point $\mathbf{d}$, a bounding box $\mathcal{B}$ centered at the initial position of $\mathbf{d}$ and a relative-error tolerance $t o l$.

Output. $K_{\kappa, \text { discr }}^{\left(\ell^{\star}\right)} \leftarrow$ an approximant of $K_{\kappa} \cap \mathcal{B}$ such that rel.error $\left(K_{\kappa, \text { discr }}^{\left(\ell^{\star}\right)}, K_{\kappa, \text { discr }}^{\left(\ell^{\star}-1\right)}\right)$ $\leq$ tol.

1. $K_{\kappa, \text { discr }}^{(0)} \leftarrow$ the convex polytope obtained by intersecting $\mathcal{B}$ with the half-plane family (14).

2. $\ell \leftarrow \ell+1$

3. $K_{\kappa, \text { discr }}^{(\ell)} \leftarrow$ the convex polytope obtained by intersecting $\mathcal{B}$ with the half-plane family (16).

4. if $K_{\kappa, \text { discr }}^{(\ell-1)} \neq \varnothing$ and rel.error $\left(K_{\kappa, \text { discr }}^{(\ell)}, K_{\kappa, \text { discr }}^{(\ell-1)}\right) \leq$ tol

5. then $\ell^{\star} \leftarrow \ell$

6. otherwise go to step 2

We end by illustrating a Maple implementation of the above algorithm for a pair of planar and a spatial Bézier configuration. Our implementation is based on Convex [3], a Maple package for convex geometry, which uses rational arithmetic and has been employed for materializing steps 1, 3 and 4 in the above algorithm.

Each test is documented by a set of figures and a table. The graphical output starts with a figure depicting the initial curve with its control polygon, the free control point $\mathbf{d}$, distinguished from the remaining ones by a circled bullet, and, finally, the corresponding convex domain $K_{\bullet}, \bullet=\kappa / \tau$, colored gray. The ensuing figures depict $K_{\bullet}$ and $K_{\bullet, \text { discr }}^{(\ell)}$, for different iteration levels, their boundaries being marked with dashed and solid line-type, respectively. The accompanying table provides, again for different iteration levels, the absolute and relative error, evaluated via (19) and (20), respectively. The first-column data correspond to the first occurrence for which $K_{\bullet}^{(\ell)}$, discr is non-empty and, as a consequence, both the absolute and the relative error cannot be 
Table 1 Intermediate values for the cubic planar Bézier curve in Fig. 1

\begin{tabular}{llllll}
\hline Order $l$ & 0 & 1 & 2 & 3 & 5 \\
\hline Abs.error & - & 2.8657 & 0.29590 & 0.11557 & $0.63280 \mathrm{e}-2$ \\
Rel.error & - & $0.34948 \mathrm{e}-1$ & $0.34866 \mathrm{e}-2$ & $0.13571 \mathrm{e}-2$ & $0.74175 \mathrm{e}-4$ \\
\# inequalities & 4 & 8 & 16 & 32 & 128 \\
\# vertices & 5 & 6 & 7 & 9 & 23 \\
CPI time (s) & $0.15 \mathrm{e}-1$ & $0.15 \mathrm{e}-1$ & $0.15 \mathrm{e}-1$ & $0.15 \mathrm{e}-1$ & $0.46 \mathrm{e}-1$ \\
\hline
\end{tabular}

The axes-aligned bounding box $\mathcal{B}$ is centered at $\mathbf{d}=(0,0)$ and has edge length equal to 20 units

Table 2 Intermediate values for the quintic planar Bézier curve in Fig. 3

\begin{tabular}{llllll}
\hline Order $l$ & 1 & 2 & 3 & 5 & 7 \\
\hline Abs.error & - & 1406.8 & 145.33 & 24.836 & 1.3061 \\
Rel.error & - & 7.1681 & $0.90659 \mathrm{e}-1$ & $0.13803 \mathrm{e}-1$ & $0.71275 \mathrm{e}-3$ \\
\# inequalities & 16 & 32 & 64 & 256 & 1024 \\
\# vertices & 6 & 4 & 4 & 3 & 3 \\
CPU time (s) & $0.15 \mathrm{e}-1$ & $0.15 \mathrm{e}-1$ & $0.31 \mathrm{e}-1$ & 0.265 & 2.839 \\
\hline
\end{tabular}

The axes-aligned bounding box $\mathcal{B}$ is chosen so that it contains the entire $K_{\kappa}$

Table 3 Intermediate values for the quintic spatial Bézier curve in Fig. 5

\begin{tabular}{llllll}
\hline Order $l$ & 0 & 2 & 3 & 4 & 5 \\
\hline Abs.error & - & 27837.0 & 3378.8 & 1029.7 & 244.36 \\
Rel.error & - & $0.16838 \mathrm{e}-1$ & $0.20099 \mathrm{e}-2$ & $0.61131 \mathrm{e}-3$ & $0.14498 \mathrm{e}-3$ \\
\# inequalities & 10 & 40 & 80 & 160 & 320 \\
\# faces & 10 & 17 & 28 & 49 & 92 \\
CPU time (s) & $0.15 \mathrm{e}-1$ & $0.62 \mathrm{e}-1$ & 0.218 & 0.889 & 5.054 \\
\hline
\end{tabular}

The axes-aligned bounding box $\mathcal{B}$ is centered at $\mathbf{d}=(0,0)$ and has edge length equal to 200 units

defined. Furthermore, the table supplies the number of inequalities used for defining the approximating polyhedron $K_{\bullet}^{(\ell)}$ discr , the number of its vertices/faces and, finally, the cpu time spent for each iteration on a typical laptop with the following processor: Intel(R) Core(TM)2 Duo CPU T7500 at $2.2 \mathrm{GHz}$ (Figs. 1, 2, 3, 4, 5, 6; Tables 1, 2, 3).

\section{References}

1. Elber G (1992) Free form surface analysis using a hybrid of symbolic and numeric computation, Ph.D. thesis, University of Utah, Computer Science Department

2. Farin G (2002) Curves and surfaces for CAGD. A practical guide, 5th edn. Morgan Kaufmann Publishers, San Francisco

3. Franz M (2006) Convex-a Maple package for convex geometry. http://www-fourier.ujf-grenoble.fr/ $\sim$ franz/convex

4. Gruber P (1988) Volume approximation of convex bodies by inscribed polytopes. Math Ann 281:229245

5. Karousos EI, Ginnis AI, Kaklis PD (2007) Quantifying the effect of a control point on the sign of curvature. Computing 79:249-259 
6. Karousos EI, Ginnis AI, Kaklis PD (2008) Controlling torsion sign. Advances in geometric modeling and processing. Lecture notes in computer science (LNCS), vol 4975, pp 92-106

7. Lyche T, Moerken K (2004) Spline methods. Draft, Department of Informatics, University of Oslo. http://heim.ifi.uio.no/knutm/komp04.pdf

8. Moerken K (1991) Some identities for products and degree raising of splines. Constr Approx 7:195-208 